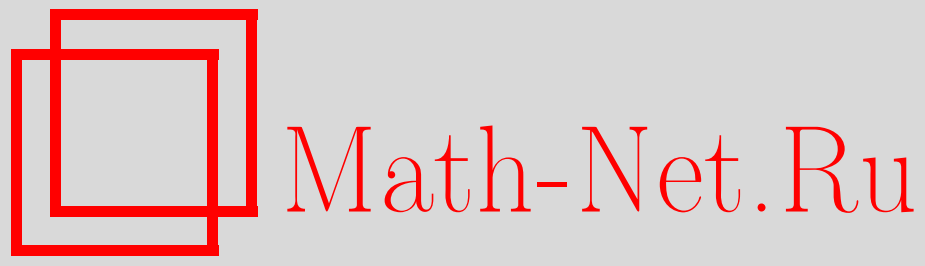

А. И. Штерн, Предсопряженные к алгебрам фон Неймана, Функи. анализ и его прил., 2003, том 37, выпуск 2, 92-94

DOI: https://doi.org/10.4213/faa153

Использование Общероссийского математического портала Math-Net.Ru подразумевает, что вы прочитали и согласны с пользовательским соглашением

http://www.mathnet.ru/rus/agreement

Параметры загрузки:

IP : 54.81 .137 .203

26 апреля 2023 г., 15:41:01

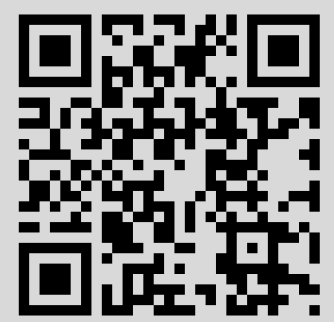


УДК 517.98

\title{
Предсопряженные к алгебрам фон Неймана
}

\author{
(C) 2003. А. И. ШТЕРН
}

В 1953 г. Диксмье [3] обнаружил, что предсопряженное пространство к алгебре фон Неймана, т. е. банахово пространство, к которому алгебра фон Неймана сопряжена, определено однозначно с точностью до изометрического изоморфизма банаховых пространств. С другой стороны, хорошо известно, что пространство $m=l^{\infty}$ ограниченных последовательностей изометрически изоморфно и второму сопряженному к пространству $c$ ограниченных последовательностей, и второму сопряженному к $\left(C^{*}\right.$-)тензорному квадрату $c \otimes c$, в то время как $c$ и $c \otimes c$ не являются изометрически изоморфными. Оказывается, неединственность второго предсопряженного к алгебре фон Неймана может иметь место, только если порядок сопряженности алгебры фон Неймана не слишком высок. А именно, справедливо следующее утверждение, часть которого была ранее опубликована в [16].

Теорема 1. (1) Пусть $\mathfrak{A}$ - алгебра фон Неймана, являющаяся третьим сопряженным (как банахово пространство) $к$ некоторому банахову пространству $A_{*}$. Тогда пространство $A=\left(A_{*}\right)^{*}$, сопряженное $\kappa A_{*}$, изометрически изоморфно алгебре фон Неймана.

(2) Если $A_{1}$ и $A_{2}$ - такие алгебры фон Неймана, ито их вторые сопряженнысе $\mathfrak{A}_{1}$ и $\mathfrak{A}_{2}$ изоморфны как алгебры фон Неймана, то $A_{1}$ и $A_{2}$ изоморфны как алгебры фон Неймана.

(3) Если $A_{1}$ и $A_{2}$ - такие алгебры фон Неймана, ито их вторые сопряженные изометрически изоморфны как банаховы пространства, то $A_{1}$ и $A_{2}$ изометрически изоморфны как банаховы пространства.

Эта теорема является новой даже в коммутативном случае. Любопытна геометрическая специфика теоремы единственности. Во многих случаях единственность предсопряженного пространства сопровождается единственностью проектора с единичной нормой на образ исходного пространства в его втором сопряженном $[14,17,19]$. В рассматриваемом случае это далеко не так. Согласно [14], существует по крайней мере континуум попарно различных проекторов с единичной нормой во втором сопряженном пространстве к алгебре фон Неймана $L^{\infty}[0,1]$ на канонический образ пространства $L^{\infty}[0,1]$ в его втором сопряженном.

Наметим доказательство теоремы 1. (1) Оператор, являющийся вторым сопряженным к каноническому проектору пространства $\mathfrak{A}$ на $A$ (этот оператор, конечно, является проектором, но не каноническим проектором), проектирует пятое сопряженное к $A_{*}$ на третье, т. е. $\mathfrak{A}^{* *}$ на его подалгебру фон Неймана $\mathfrak{A}$. Кроме того, он унитален (см. доказательство теоремы 3.4 из [15, Ch. III, §3]). Поэтому он вполне положителен [11], и его ограничение на канонический образ алгебры $\mathfrak{A}$ в $\mathfrak{A}^{* *}$, являющееся каноническим проектором пространства $\mathfrak{A}$ на $A$, тоже вполне положительно и унитально. Следовательно, в $A$ можно ввести умножение, превращающее $A$ в $C^{*}$-алгебру $[6,11]$, которая является $W^{*}$-алгеброй, потому что ее банахово пространство - сопряженное. Это улучшает доказательство существования, набросок которого дан в [16].

(2) Отождествим изоморфные как алгебры фон Неймана обертывающие алгебры $\mathfrak{A}_{1}$ и $\mathfrak{A}_{2}$ и обозначим эту алгебру фон Неймана через $\mathfrak{A}$. Для доказательства 
единственности напомним [1, Théorème 11$]$, что $\mathfrak{A}$ есть прямая сумма канонического образа алгебры $A_{1}$ в $\mathfrak{A}$ и ядра канонического гомоморфизма алгебры $\mathfrak{A}$ на $A_{2}[9,12.1 .6]$. Следовательно, ограничение этого канонического гомоморфизма на канонический образ алгебры $A_{1}$ в $\mathfrak{A}$ определяет гомоморфизм алгебры фон Неймана $A_{1}$ на алгебру фон Неймана $A_{2}$ с тривиальным ядром. Такой гомоморфизм является алгебраическим изоморфизмом. Поскольку любой изоморфизм алгебр фон Неймана изометричен [8, p. 9], мы видим, что алгебры фон Неймана $A_{1}$ и $A_{2}$ изометрически изоморфны.

(3) Пусть $A_{1}$ и $A_{2}$ - такие алгебры фон Неймана, что их вторые сопряженные изометрически изоморфны как банаховы пространства. При изометрическом изоморфизме банаховых пространств $A_{1}^{* *}$ и $A_{2}^{* *}$ канонический образ алгебры фон Неймана $A_{2}$ в $A_{2}^{* *}$ переходит в некоторую $C^{*}$-подалгебру. Изменяя при необходимости умножение в $A_{2}^{* *}$, мы получим изоморфные алгебры фон Неймана $A_{1}^{* *}$ и $A_{2}^{* *}$, а при изменении умножения, сохраняющем метрику, любая $C^{*}$-подалгебра переходит снова в $C^{*}$-подалгебру [2]. Согласно уже доказанному утверждению $(2), C^{*}$-подалгебра, соответствующая каноническому образу алгебры $A_{2}$, изоморфна (как алгебра фон Неймана) алгебре фон Неймана $A_{1}$ (точнее, ее каноническому образу в $\left.A_{1}^{* *}\right)$; в частности, эта подалгебра изометрична $A_{1}$ как банахово пространство. Таким образом, $A_{1}$ и $A_{2}$ изометрически изоморфны как банаховы пространства, что завершает доказательство.

Заметим, что алгебра фон Неймана, являющаяся третьим сопряженным (как банахово пространство) к некоторому банахову пространству, может не быть обертывающей алгеброй фон Неймана ни для какой $C^{*}$-алгебры. Примером может служить обертывающая алгебра фон Неймана фактора фон Неймана, не антиизоморфного себе [12], в которой умножение в каноническом образе фактора заменено на противоположное, а в наросте - сохранено.

Теорема 1 позволяет упростить (в своем первоначальном виде - довольно громоздкое) доказательство следующего утверждения, которое было известно ранее только в коммутативном случае [13], т. е. для $M$ - и $L$-пространств (определения этих классов пространств можно найти в $[7,10])$.

Теорема 2. Бесконечномерная алгебра фон Неймана не может иметь предсопряженные пространства любого порядка. Таким образом, для любой алгебры фон Неймана $А$ однозначно определено такое натуральное число $n$, что $А$ является $n$-м сопряженным $к$ некоторому банахову пространству, но не существует банахова пространства, для которого $A$ является $(n+1)-м$ сопряженным.

Последовательность предсопряженных пространств к $A$, о которой идет речь в теореме 2, не определена однозначно, но различия могут быть только в первом элементе цепочки (расположенной в порядке сопряженности), и только если этот элемент - пространство, второе сопряженное к которому изометрически изоморфно алгебре фон Неймана, но которое само не является сопряженным пространством. Класс таких пространств (о некоторых свойствах этого класса можно узнать из теоремы 3, см. ниже) является некоммутативным аналогом известного класса пространств Линденштрауса.

Доказательство теоремы 2 связано с неравенством для размерности центра алгебры фон Неймана и ее обертывающей алгебры. А именно, если центр алгебры фон Неймана бесконечномерен, то размерность центра обертывающей алгебры строго больше размерности центра исходной алгебры (ср. [13]), а убывающая 
цепочка порядковых чисел неизбежно конечна. Если же центр алгебры фон Неймана конечномерен, то она является прямой суммой факторов и пространство, предсопряженное к такой алгебре, является конечной $l^{1}$-суммой предсопряженных пространств к слагаемым. Можно воспользоваться следующим утверждением, которое входило в (оставшееся неопубликованным) доказательство основного утверждения заметки [16] и было приведено в [18].

Теорема 3. Пусть А - алгебра фон Неймана, и пусть ч - крайняя точка пространства нормальных состояний алгебры фон Неймана А. Тогда $\varphi-$ чистое состояние, т.е. крайняя точка пространства всех состояний алгебры фон Неймана А. В частности, если A - алгебра фон Неймана, являющаяся вторым сопряженным пространством (как банахово пространство), то А содержит минимальные проекторы и имеет нетривиальное нормальное неприводимое *-представление (и тем более имеет ненулевое прямое слагаемoe muna I).

Теорема 3 доказывается прямой проверкой всех ее утверждений; основным средством является разложение состояний на алгебре фон Неймана на нормальную и сингулярную части.

Завершение доказательства теоремы 2 теперь очевидно: алгебра фон Неймана с конечномерным центром является вторым сопряженным тогда и только тогда, когда она принадлежит типу I, и является третьим сопряженным пространством к банахову пространству тогда и только тогда, когда эта алгебра конечномерна (поскольку пространство операторов со следом в бесконечномерном гильбертовом пространстве не является алгеброй фон Неймана). В частности, получаем

СледствиЕ. Фактор фон Неймана, являющийся вторым сопряженным как банахово пространство, является фактором типа I.

В классических работах Такеды [4] и Гротендика [5] дополнительно предполагалось, что рассматриваемый фактор есть второе сопряженное к $C^{*}$-алгебре.

Результаты заметки подробно изложены в $[20,21]$.

\section{ЛИТЕРАТУРА}

1. Dixmier J. Duke Math. J, 15, 1057-1071 (1948). 2. Kadison R. V. Ann. of Math., 54, 325-338 (1951). 3. Dixmier J. Bull. Soc. Math. France, 81, 9-39 (1953). 4. Takeda Z. Tôhoku Math. J., 6, 212-219 (1954). 5. Grothendieck A. J. Math. Pures Appl., 36, Fasc. 2, 97-108 (1957). 6. Tomiyama J. Proc. Japan Acad., 33, 608-612 (1957). 7. Данфорд H., Швари Дж. Т. Линейные операторы. Т. І. ИЛ, М., 1962. 8. Dixmier J. Les algèbres d'opérateurs dans l'espace hilbertien. Gauthier-Villars, Paris, 1969. 9. Диксмье Ж. С ${ }^{*}$-алгебры и их представления. Наука, M., 1974. 10. Шефер $X$. Топологические векторные пространства. Мир, М., 1971. 11. Choi M.-D., Effros E. J. Funct. Anal., 24, 156-209 (1974). 12. Connes A. Ann. Math. (2), 101, 536-554 (1975). 13. Armstrong T. E. Proc. Amer. Math. Soc., 74, 285-290 (1979). 14. Godefroy G. Ann. Inst. Fourier, Grenoble, 29, No. 4, 53-70 (1979). 15. Takesaki M. Theory of Operator Algebras I. Springer-Verlag, New York-Heidelberg-Berlin, 1979. 16. Штерн А. И. Функц. анализ и его прил., 16, вып. 2, 90 (1982). 17. Godefroy G. Ann. scient. Éc. Norm. Sup. 4 e série, 16, 109-122 (1983). 18. Chu C. H. In: Operator algebras and operator theory (Craiova, 1989) (Pitman Res. Notes Math. Ser., 271), Longman Sci. Tech., Harlow, 1992, pp. 62-68. 19. Ng P. W., Ozawa N. Pacific J. Math., 205, 171-195 (2002). 20. Shtern A. I. Russian J. Math. Phys., 10, No. 1, 124-127 (2003). 21. Shtern A. I. Adv. Stud. Contemp. Math., 7, No. 1 (2003). 